\title{
THE DYNAMICS AND CONTROL OF AN MOVING MASS TRAVELING ON AN INITIALLY CURVED BEAM
}

\section{Chih-Ching Chang}

Graduate Student, Department of Mechatronics Engineering, National Chunghua University of Education, Chunghua, Taiwan, R.O.C.

\section{Yi-Ming Wang}

Professor, Department of Mechatronics Engineering, National Chunghua University of Education, Chunghua, Taiwan, R.O.C., Wangym@cc.ncue.edu.tw

\section{Recommended Citation}

Chang, Chih-Ching and Wang, Yi-Ming (2007) "THE DYNAMICS AND CONTROL OF AN MOVING MASS TRAVELING ON AN INITIALLY CURVED BEAM," Journal of Marine Science and Technology. Vol. 15: Iss. 4, Article 1.

DOI: $10.51400 / 2709-6998.2043$

Available at: https://jmstt.ntou.edu.tw/journal/vol15/iss4/1

This Research Article is brought to you for free and open access by Journal of Marine Science and Technology. It has been accepted for inclusion in Journal of Marine Science and Technology by an authorized editor of Journal of Marine Science and Technology. 


\title{
THE DYNAMICS AND CONTROL OF AN MOVING MASS TRAVELING ON AN INITIALLY CURVED BEAM
}

\author{
Chih-Ching Chang* and Yi-Ming Wang**
}

Key words: dynamic control, LQR control.

\begin{abstract}
The objective of this paper is to study the dynamics and control of an Euler-Bernoulli beam with an attached mass rolling on an initially curved beam based on a simplified control model. The optimization of the performance index of linear quadratic regulator $(\mathrm{LQR})$ is considered. Three actuators are placed along the beam that provide the necessary forces to control the response of the beam-mass system.

Based on Euler-Bernoulli beam theory and Newtonian mechanics, the coupled non-linear equations of motion combining with a simple closed-loop feedback control are obtained. Results of present study show that with appropriate regulator design, the vibration of the system can be suppressed significantly even if a simplified control model is applied.
\end{abstract}

\section{INTRODUCTION}

The dynamics and dynamic control of flexible structures with an attached moving mass have been the subjects of many studies. Ting et al. [6] discussed the problem regarding the interaction between the moving mass and the supporting structure. They concluded that if "correct" formulation is desired the convective acceleration terms should be included.

Mohamed et al. [2,3] studied the problem of active control of flexible structures under moving loads. The tendon control is used to suppress the vibration of the beam. Sung [5] studied similar problem except in his study, he employed two piezoelectric actuators that are bonded at the bottom of the beam at different locations to suppress the vibration of the beam under a moving mass.

Paper Submitted 02/15/06, Accepted 05/17/06. Author for Correspondence: Yi-Ming Wang. E-mail: Wangym@cc.ncue.edu.tw

*Graduate Student, Department of Mechatronics Engineering, National Chunghua University of Education, Chunghua, Taiwan, R.O.C.

**Professor, Department of Mechatronics Engineering, National Chunghua University of Education, Chunghua, Taiwan, R.O.C.
Wang [7] studied the transient dynamics of a beammass system carrying multiple masses moving along an initially curved beam and showed that the initial curvature of a beam can result significant effects to the dynamics of the system even if the initial imperfection of the beam is small.

Gardonio et al. [1] analyzed the controlled response of a beam with direct velocity feedback control. They found that new regions of natural frequencies and mode shapes are generated if very high control gains of the velocity feedback control are applied to the system.

The problem of moving mass has been studied by many authors, however, the dynamics and control of an attached mass rolling on an initially curved beam has not been studied yet. Hence, a beam-mass system with a geometric imperfection beam is considered. In this paper, the mechanics of the problem is Newtonian. The control law considered is based on the optimal linear quadratic regulator (LQR) method. The equations of motion combining with a simple closed-loop feedback control are derived. The dynamics of the system with and without control are studied. The effects produced by the variation of physical parameters to the response of the system are examined.

\section{MODEL FORMULATION}

In this study, a moving mass rolling on a finite, simple supported, initially curved beam is considered. The beam rests on a uniform elastic foundation and is of length $\ell$ and initial variation $\bar{v}_{0}(s)$. Here $\bar{v}_{0}(s), \bar{v}_{0}(s)=v_{0}^{*}$ $\sin \frac{\pi s}{\ell}$, is the initial deviation of the beam measured from straight axis with $v_{0}^{*}$ being the amplitude of initial deviation.

From Figure 1, the equations governing the motion of the system can be derived from the dynamic equilibrium of forces and momenta and are given as

$$
\begin{aligned}
& \left\{\left[T \cos \left(\theta+\theta_{0}\right)-V \sin \left(\theta+\theta_{0}\right)\right] \mathbf{i}\right. \\
& \left.\quad+\left[T \cos \left(\theta+\theta_{0}\right)+V \sin \left(\theta+\theta_{0}\right)\right] \mathbf{j}\right\},{ }_{s}+\mathbf{f}
\end{aligned}
$$




$$
\begin{aligned}
\approx & \left\{\left[T+E I v_{s s s}\left(v_{, s}+\bar{v}_{0, s}\right)\right] \mathbf{i}+\left[T\left(v_{, s}+\bar{v}_{0, s}\right)\right.\right. \\
& \left.\left.-E I v,,_{s s s}\right] \mathbf{j}\right\},_{s}+\mathbf{f}=m \mathbf{r},,_{t t} \quad 0<s<l, t>0
\end{aligned}
$$

with the inextensibility constraint $\mathbf{r},_{\mathrm{s}} \cdot \mathbf{r},_{\mathrm{s}}=1$. In the above equation, $\mathbf{i}$ and $\mathbf{j}$ represent the unit vectors of the coordinate system in the $x$ and $y$ directions, respectively; $\mathrm{v}(s, t)$ is the transverse displacements of the beam from the undeformed state; $T, V, m, E$, and $I$ are the axial force in the beam, the transverse force in the beam, the mass per unit length of the beam, the Young's modulus, and the area moment of inertia of the beam, respectively; the subscript $s$ and $t$ denote the $s$ and $t$ differentiation. $\mathbf{r}(s, t)$ is the Cartesian position vector of point $\mathrm{s}$ along the beam at time $t$. The force $\mathbf{f}$ denotes the external forces including the weight of the moving mass, the moving reaction of the mass upon the beam, and the control force applied and is given as

$$
\mathbf{f}=-\left(k \mathrm{v}+u_{k}(t) \delta\left(s-s_{k}\right)\right) \mathbf{j}+(N \mathbf{n}+\mu N \hat{\boldsymbol{\tau}}) \delta(s-\bar{s})
$$

where $\bar{s}(t)$ is the distance along the arc of the beam designating the position of the moving mass; $\delta(s-\bar{s})$ and $u_{k}(t)$ are Dirac delta function and control force of the $k^{t h}$ actuator at the location $s_{k}$, respectively; $\hat{\tau} \approx \mathbf{i}+$ $\left(v_{, \mathrm{s}}+\bar{v}_{0, s}\right) \mathbf{j}$ and $\mathbf{n} \approx-\left(v_{\mathrm{s}}+\bar{v}_{0, s}\right) \mathbf{i}+\mathbf{j}$.

The equation of motion of the moving mass obeys

$$
M \mathbf{a}_{M}=M g+\mathbf{f}_{m}-\mu N \hat{\tau}-N \mathbf{n}
$$

where $M=$ mass of the moving mass, $\mathbf{a}_{M}=\frac{d^{2}}{d t^{2}}[\mathbf{r}(\bar{s}(t), t)]$, $\mathbf{g}=\mathrm{g} \mathbf{j}, \mathbf{f}_{\mathrm{m}}=$ tangential propelling force with $\mathrm{f}$ being a prescribed function of time $=M f \hat{\tau}, N=$ reaction of beam on the mass, and $\mu=$ coefficient of friction,

In the following, the attached mass moves along the beam with constant velocity and the condition of

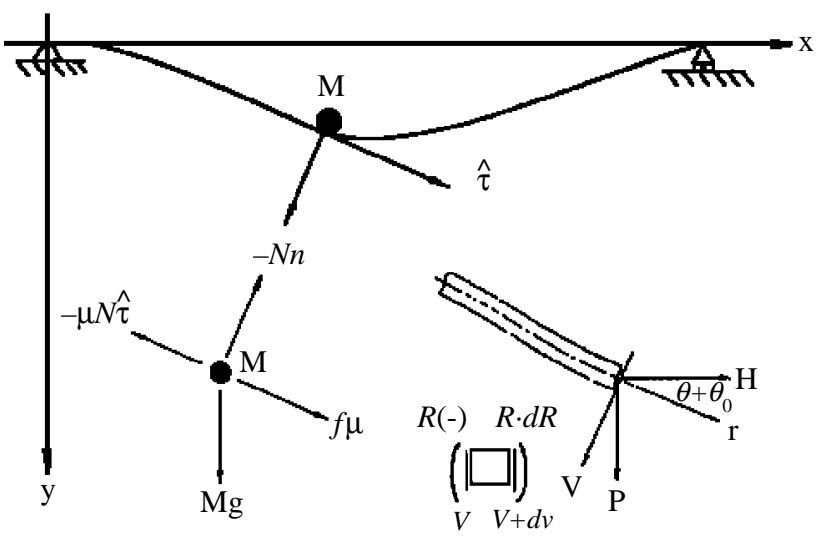

Fig. 1. System configuration and force equilibrium diagram. small deformations are assumed. For this, one sets $\mathbf{a}_{M}$ $=\mathbf{0}$ and substitutes Eq. (3) into Eq. (2) to eliminate the term $\mu N \hat{\tau}, N \mathbf{n}$. For the second assumption, one neglects nonlinear terms when compares these terms to the linear term in the displacement field and unity. In addition, the axial force $T$ can be determined by integrating equation (1) in $\mathbf{i}$ direction under the assumption that the variation of axial force is assumed to remain constant at the moving mass and uses the boundary condition in $\mathbf{i}$ direction. After some manipulations, it is found that the axial force $T$ is in the second-order of nonlinearity and can be neglected.

By introducing the following dimensionless quantites

$$
\begin{aligned}
& \tau=\sqrt{\frac{E I}{m l^{4}} t}, \hat{M}=\frac{M}{m l}, \hat{f}=\frac{m l^{3}}{E I} f, \hat{g}=\frac{m l^{3}}{E I} g, \\
& \hat{k}=\frac{k l^{3}}{E I}, \eta=\frac{s}{l}, \xi=\frac{\bar{s}}{l}, \hat{\mathrm{v}}=\frac{v}{l}, \hat{\bar{v}}_{0}=\frac{\bar{v}_{0}}{l}
\end{aligned}
$$

the equation of motion associated with the boundary conditions in $\mathbf{j}$ direction can be obtained. By representing $\hat{v}$ as a continuous function, $\hat{v}=\sum_{n=1}^{\infty} A_{n}(\tau) \sin n \pi \eta, 0<\eta$ $\langle 1, \tau\rangle 0$, such that the simple supported boundary condition in $\mathbf{j}$ direction is satisfied, the approximate solution of the beam-mass system then can be obtained by employing the Galerkin's method. Using Galerkin's procedure for minimizing error, one obtains

$$
\begin{aligned}
\ddot{A}_{j}(\tau) & +\omega_{j}^{2} A_{j}(\tau)=2 \hat{M}\left\{\hat{g} \hat{S}_{j}(\xi)\right. \\
& +\mu \hat{g} \sum_{n=1}^{\infty} R_{j n}(\xi) v_{0} A_{n}(\tau)+(\xi)^{2} S_{j 1}(\xi) v_{0} \\
& -\sum_{n=1}^{\infty}\left[\hat{S}_{j n}(\xi) \ddot{A}_{n}(\tau)+2 \xi R_{j n}(\xi) \dot{A}_{n}(\tau)\right. \\
& \left.\left.-(\xi)^{2} S_{j n}(\xi) A_{n}(\tau)\right]\right\}-\sum_{k=1}^{r} \hat{S}_{j}\left(\eta_{k}\right) \hat{u}_{k}, \\
& 0<\eta<1, \tau>0
\end{aligned}
$$

where $\hat{u}_{k}=\frac{u_{k} l^{3}}{E I}, v_{0}=\frac{v_{0}^{*}}{l}, \omega_{j}^{2}=\left((j \pi)^{4}+\hat{k}\right)$,

$\hat{\bar{v}}_{0}=\frac{v_{0}}{\ell}=\frac{v_{0}^{*}}{\ell} \sin \pi \eta \equiv v_{0} \sin \pi \eta$,

$R_{j n}(\xi)=(n \pi) \cos n \pi \xi \sin j \pi \xi$,

$S_{j n}(\xi)=(n \pi)^{2} \sin n \pi \xi \sin j \pi \xi \equiv(n \pi)^{2} \hat{S}_{j n}(\xi)$,

$\hat{S}_{n}(\xi)=\sin n \pi \xi, \hat{S}_{j}\left(\eta_{k}\right)=\sin j \pi \eta_{k}$.

To write the equation of motion in matrix form, it allows the parameters $j$ and $n$ to have the range from 1 to $N$; the parameter $k$ has the range from 1 to $r$. Let $\mathrm{y}=$ 
$\left(A_{1}, A_{2}, \ldots . ., A_{N}\right)^{T}$, then, Eq. (5) can be written as

$$
\mathbf{M}(\xi) \ddot{\mathbf{y}}(\tau)+\mathbf{K}_{1}(\xi) \dot{\mathbf{y}}(\tau)+\mathbf{K}_{2}(\xi) \mathbf{y}(\tau)=\overline{\mathbf{h}}(\xi)+\overline{\mathbf{B}} \overline{\mathbf{u}}
$$

The initial conditions are

$$
\mathbf{y}\left(t_{0}\right)=\mathbf{y}_{0}, \dot{\mathbf{y}}\left(t_{0}\right)=\dot{\mathbf{y}}_{0}, \dot{\xi}\left(t_{0}\right)=\mathrm{V}_{\xi}, \xi\left(t_{0}\right)=\mathrm{V}_{\xi} t_{0}
$$

where $\dot{\xi}\left(t_{0}\right)$ and $\xi\left(t_{0}\right)$ are the initial speed and position of the mass along the beam at time $t_{0}$, respectively. The components of the previously defined matrices, vectors and scalars in Eq. (6), i.e., $\mathbf{M}, \mathbf{K}_{1}, \mathbf{K}_{2}, \overline{\mathbf{h}}, \overline{\mathbf{B}}$ and $\overline{\mathbf{u}}$ are given in the Appendix.

Introducing new state vectors $\mathbf{z}$ into Eq. (6) to obtain the numerical integration scheme of the system with the associated initial conditions as specified in Eq. (7), let $\mathbf{z}=\left(\dot{\mathbf{y}}^{T}, \mathbf{y}^{T}\right)$, where $\mathbf{z}$ is the $2 N$ vector. The initial condition of $\mathbf{z}$ is $\mathbf{z}\left(t_{0}\right)=\left(\dot{\mathbf{y}}_{0}^{T}, \mathbf{y}_{0}^{T}\right)$, then the Eq. (6) can be written as

$$
\tilde{\mathbf{M}} \dot{\mathbf{z}}+\tilde{\mathbf{N}} \mathbf{z}=\hat{\mathbf{B}} \mathbf{u}+\mathbf{h}
$$

or

$$
\dot{\mathbf{z}}=\mathbf{A z}+\mathbf{B u}+\mathbf{d}
$$

where $\mathbf{A}=-\tilde{\mathbf{M}}^{-1} \tilde{\mathbf{N}}, \mathbf{B}=\tilde{\mathbf{M}}^{-1} \hat{\mathbf{B}}, \mathbf{d}=\tilde{\mathbf{M}}^{-1} \mathbf{h}$. In addition,

$$
\begin{aligned}
& \tilde{\mathbf{M}}=\left[\begin{array}{cc}
\mathbf{M} & {[0]} \\
{[\mathbf{0}]} & \mathbf{I}
\end{array}\right], \tilde{\mathbf{N}}=\left[\begin{array}{ll}
\mathbf{K}_{\mathbf{1}} & \mathbf{K}_{2} \\
-\mathbf{I} & {[\mathbf{0}]}
\end{array}\right], \mathbf{h}=\left(\overline{\mathbf{h}}^{\mathbf{T}}, \mathbf{0}^{\mathbf{T}}\right) \\
& \hat{\mathbf{B}}=\left[\begin{array}{cc}
\overline{\mathbf{B}} & {[0]_{n-r}} \\
{[0]_{\mathbf{r}}} & {[0]_{n-r}}
\end{array}\right], \mathbf{u}=\left(\overline{\mathbf{u}}^{T}, \mathbf{0}_{n-r}^{T}\right)^{T}
\end{aligned}
$$

where $\mathbf{A}=2 N \times 2 N$ matrix,

$\mathbf{B}=2 N \times 2 N$ matrix,

$\mathbf{u}=(N+r) \times(N \times(N-r)+1)$ matrix,

$\mathbf{d}=2 N \times 1$ matrix, $\mathbf{I}=N \times N$ unit matrix,

$\overline{\mathbf{B}}_{r}=N \times r$ matrix, [0] $=N \times N$ matrix,

$[0]_{r}=N \times r$ matrix, $\overline{\mathbf{u}}_{r}=r \times 1$ matrix,

$[\mathbf{0}]_{n-r}=N \times(N \times r)$ matrix.

\section{OPTIMAL CONTROL DESIGN}

\section{Basic formulas}

The linear quadratic regulator optimal control is employed to obtain suitable control gains for the beammass system. The performance index is

$J=\frac{1}{2} \mathbf{z}^{T}\left(t_{f}\right) \overline{\mathbf{H}} \mathbf{z}\left(t_{f}\right)+\frac{1}{2}\left\{\int_{t_{0}}^{t_{f}}\left[\mathbf{z}^{T}(t) \mathbf{Q}(t) \mathbf{z}(t)\right.\right.$

$$
\left.\left.+\mathbf{u}^{T}(t) \mathbf{R}(t) \mathbf{u}(t)\right] d t\right\}
$$

where the state weighting matrices $\overline{\mathbf{H}}, \mathbf{Q}$ and $\mathbf{R}$ are symmetric semi-definite and positive-definite matrices, respectively; $t_{0}$ and $t_{f}$ are the initial and final time of the control interval. The control input is given by (Shahian et al., 1993).

$$
\mathbf{u}^{*}(t)=-\mathbf{R}^{-1} \mathbf{B}^{T} \mathbf{P}(t) \mathbf{z}(t)
$$

where $\mathbf{R}^{-1} \mathbf{B}^{T} \mathbf{P}(t)$ is the optimal control gain of the state by minimizing Eq. (10) subjected to the constraint, Eq. (9). Here, $\mathbf{P}(t)$ is a self-adjoint, positive definite Riccati matrix satisfying the nonlinear differential equation.

$$
-\dot{\mathbf{P}}=\mathbf{P A}+\mathbf{A}^{T} \mathbf{P}-\mathbf{P B R}^{-1} \mathbf{B}^{T} \mathbf{P}+\mathbf{Q}
$$

The controlled system is then expressed as

$$
\dot{\mathbf{z}}=\left[\mathbf{A}-\mathbf{B} \mathbf{R}^{-1} \mathbf{B}^{T} \mathbf{P}\right] \mathbf{z}+\mathbf{d}
$$

The corresponding block diagram of the closedloop system governed by Eq. (9) is given in Figure 2.

\section{Actuators placement}

It is known that the placement of actuators plays a major role in optimal active control of flexible structures. In order to maximize the performance of the controlled system and minimize the number of actuators, the best locations of actuators can be chosen and achieved by iteration such that the performance index is minimized. In order to diminish the error produced by the uncontrolled modes of vibration, three actuators are selected to provide better control behavior than the result of a single actuator. Figure 3 shows the comparison of the controlled response of the system by using a single actuator (dashed line) at the mid-span and by three actuators (solid line). It clearly indicates that the result of applying three actuators is better than the result of using a single actuator at the mid-span.

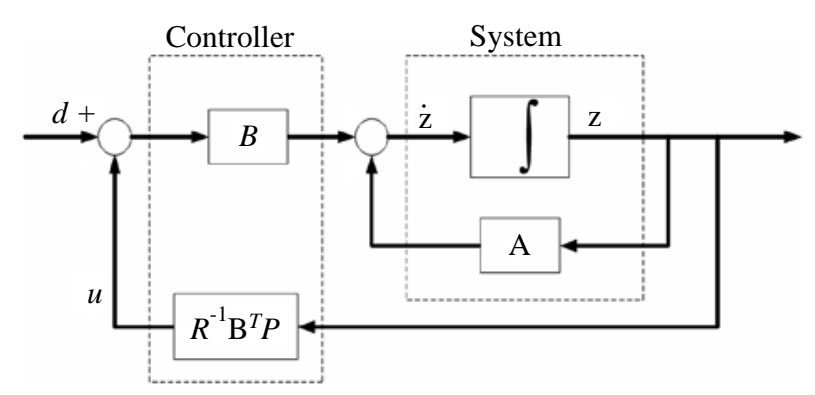

Fig. 2. Block diagram of optimal feedback control. 


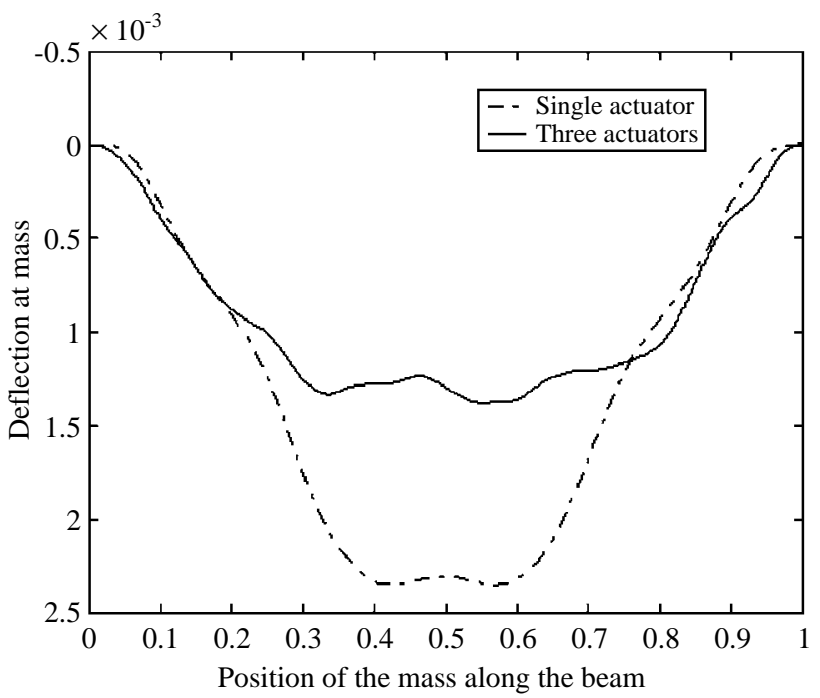

Fig. 3. The Deflection at mass vs. position of mass along the beam due to the influence of single actuator and three actuators.

\section{NUMERICAL SIMULATION}

As mentioned previously, three actuators attached on the beam at different locations are sufficient to provide the required control force to the system. After some manipulations, it is found that the locations of the three actuators are at $\hat{s}_{1}=0.26, \hat{s}_{2}=0.5$ and $\hat{s}_{3}=0.82$. Other parameters, unless otherwise specified, are: EI/ $m l^{4}=(9.4 / \pi)^{2}, \mu=\hat{k}=0.0, \mathbf{Q}=10^{6}$, and $\mathbf{R}=1$. Figure 4 shows the control performance (deflection at midspan) vs. the values of matrix $\mathbf{Q}$ for two different values of initial speed of the mass, $\dot{\xi}(0)=0.25 \pi$ and $0.5 \pi$. This figure clearly indicates that the maximum control per-

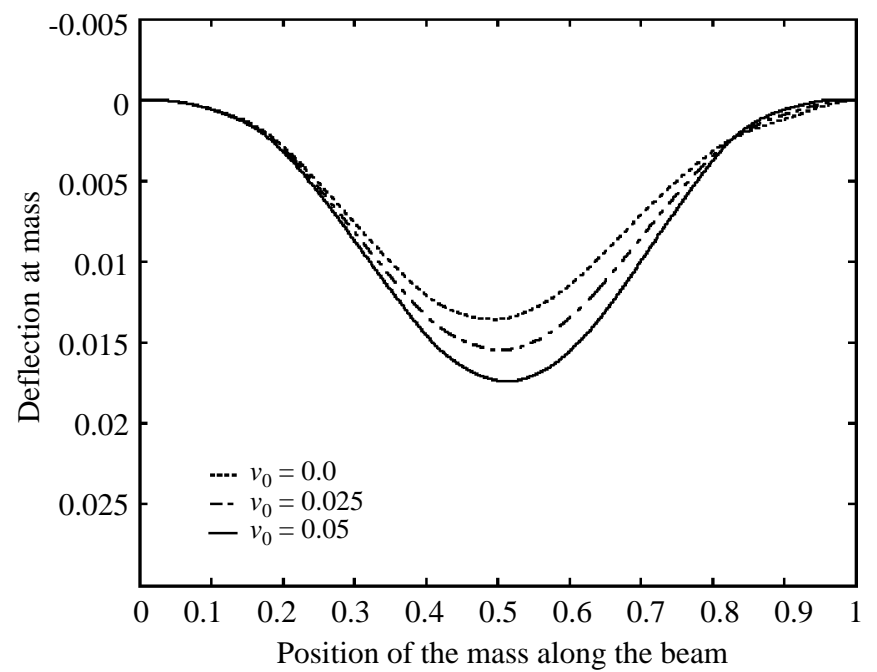

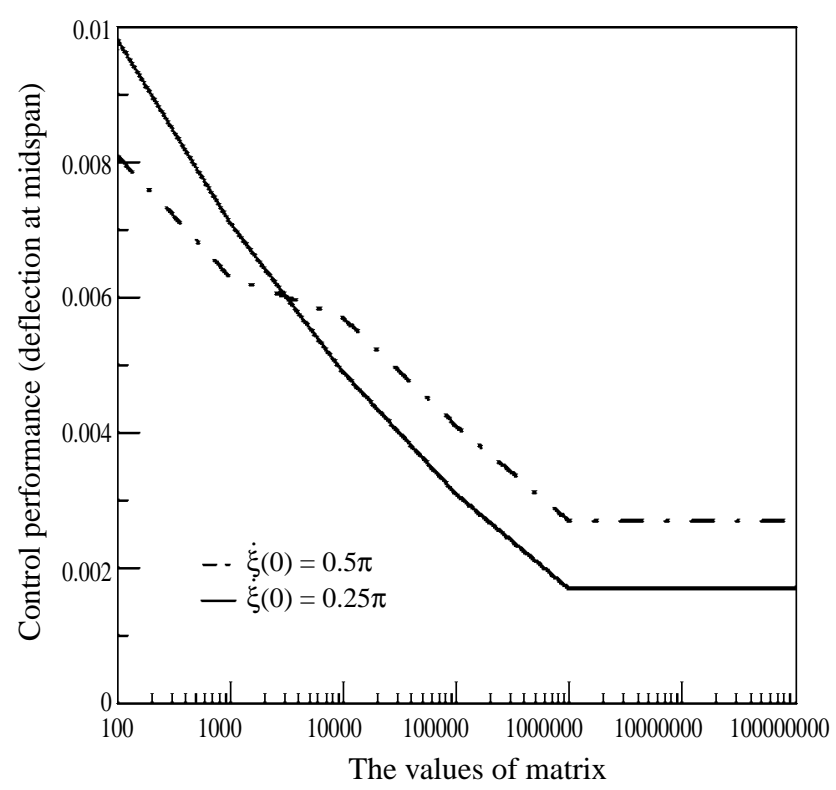

Fig. 4. Control performance (Deflection at midspan) vs. the values of matrix $Q$ for two different values of initial speed of the mass, $\dot{\xi}(0)=0.25 \pi$ and $0.5 \pi$.

formance is reached when $\mathbf{Q} \geq 10^{6}$. Hence, the value of $\mathbf{Q}$ is set to be $10^{6}$.

For numerical integration of the system, Eqs. (9) and (13), the Runge-Kutta method with sixth order accuracy is used. The accuracy of the model is checked with the results of Ting, et al. (1974). The dimension $N$ of the state vector $\mathbf{z}$ is set to be 30 to retain sufficient convergence.

Figures 5 and 6 describe the response of the system due to the motion of the moving mass without and with control under different conditions. Figure 5 shows the

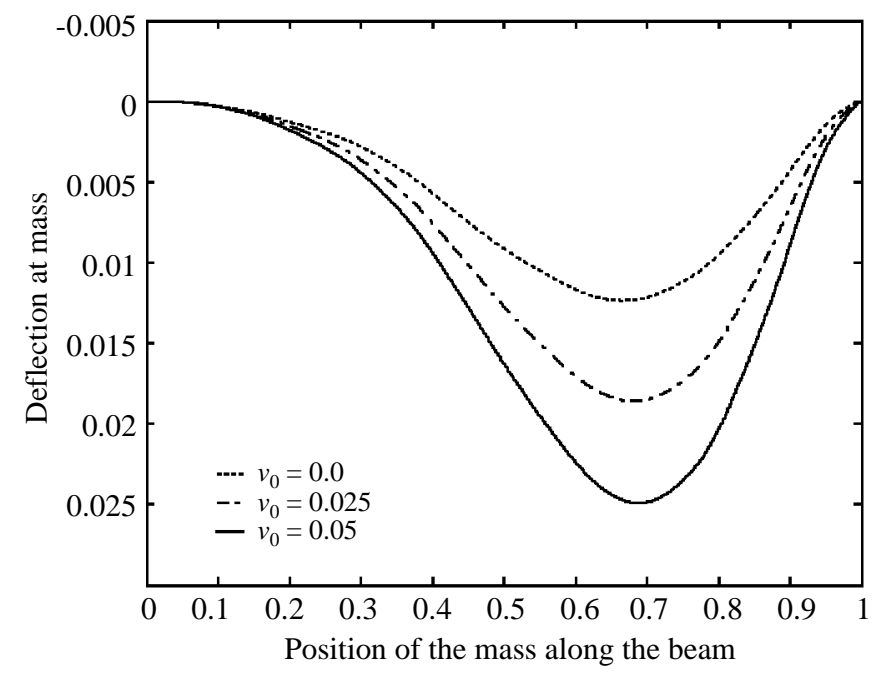

Fig. 5. Deflection at mass vs. the position of mass along the beam due to the influence geometric imperfection of beam for two different values of initial speed of the mass, $\dot{\xi}(0)=0.25 \pi$ (top plot) and $\pi$. 

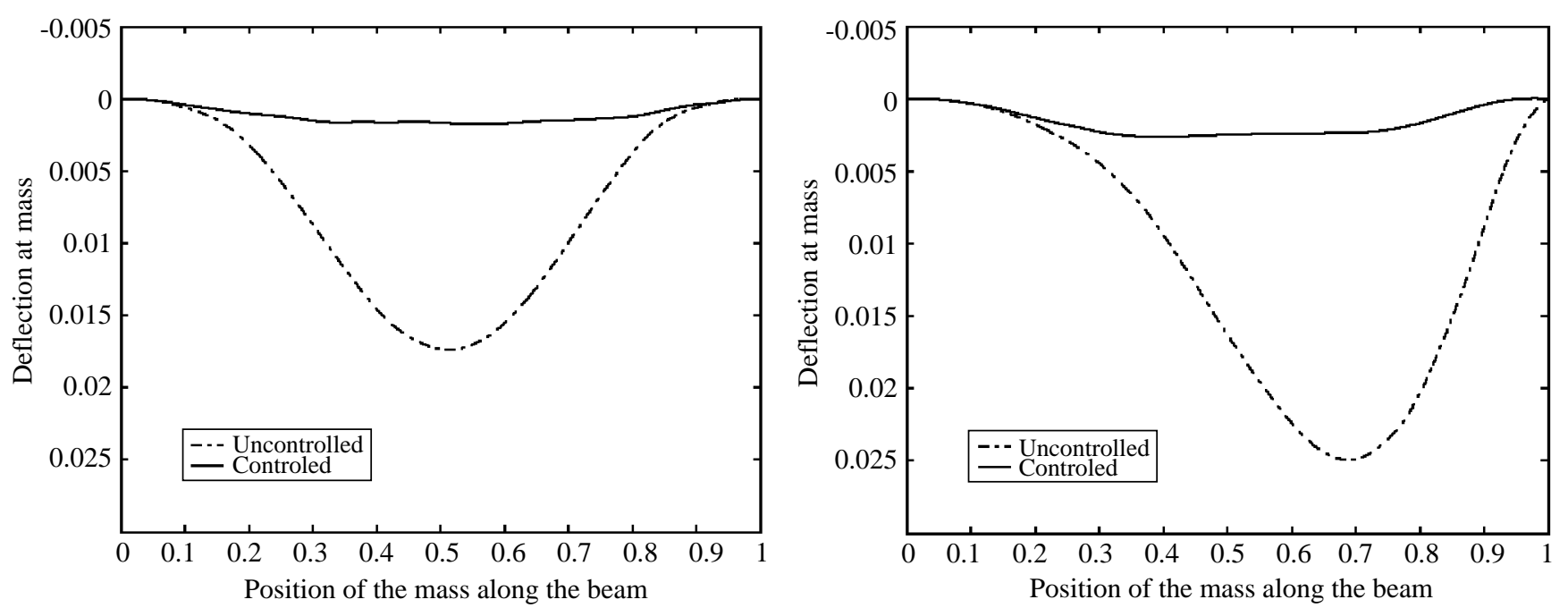

Fig. 6. The Deflection at mass vs. position of mass along the beam due to the influence of two different values of initial speed of the mass, $\dot{\zeta}(0)=0.25 \pi$ (upper plot) and (below plot); other parameters used are $\hat{M}=0.5$ and $v_{0}=0.05$.

deflection at mass (trajectory) vs. the position of mass along the beam due to the influence of geometric imperfection of beam for $\hat{M}=0.5, \dot{\xi}(0)=0.25 \pi$ (top plot), and $0.5 \pi$ (bottom plot). Three different values of initial curvature are selected; they are $v_{0}=0.0$ (dot line), $v_{0}=$ 0.025 (dash-dot line), and $v_{0}=0.05$ (solid line). This figure clearly indicates that the initial curvature of a beam plays an important role to the response of a beammass system. In general, the initial imperfection amplifies the amplitude of deflection at mass even if the initial deviation is small.

Figure 6 presents the response of the system with and without control for $v_{0}=0.05$ and $\hat{M}=0.5$. Two different values of initial speed of the moving mass are used, $\dot{\xi}(0)=0.25 \pi$ (top plot) and $0.5 \pi$ (bottom plot). It clearly shows that the vibration of the system can be efficiently suppressed by the proposed linear quadratic regulator control method even if a beam with initial geometric imperfection is considered.

\section{CONCLUSIONS}

The dynamics and control of a moving mass rolling on an initially curved beam are studied. The optimal active control theory with feedback closed-loop is applied to suppress the oscillation of the system. In order to provide necessary control forces, three actuators are attached on the beam. Results of present study show that the vibrations of the system can be suppressed significantly even if the applied control system is a simple model.

\section{REFERENCES}

1. Gardonio, P. and Elliott, S.J., "Modal Response of a
Beam with a Sensor-Actuator Pair for the Implementation of Velocity Feedback Control," Journal of Solids and Structures, Vol. 284, pp. 1-22 (2005).

2. Mohamed, A.R. and Horst, H.L., "Active Control of Flexible Structures," Journal of the Structural Division, ASCE, ST8, pp. 1251-1265 (1978).

3. Mohamed, A.R. and Horst, H.L., "Automatic Active Control of Structures," Journal of the Structural Division, ASCE, ST3, pp. 663-667 (1980).

4. Shahian, B. and Hassul, M., Control System Design Using MATLAB, Prentice Hall, Englewood Cliffs, NJ, pp. 366-394 (1993).

5. Sung, Y.G., "Modelling and Control with Piezoactuators for a Simply Supported Beam under a Moving Mass," Journal of Sound and Vibration, Vol. 250, pp. 617-626 (2002).

6. Ting, E.C., Genin, J., and Ginsberg, J.H., "A General Algorithm for Moving Mass Problems," Journal of Sound and Vibrations, Vol. 33, pp. 49-58 (1974).

7. Wang, Y.M., "The Transient Dynamics of Multiple Accelerating/Decelerating Masses Traveling on an Initially Curved Beam," Journal of Sound and Vibration, Vol. 286, pp. 207-228 (2005).

\section{APPENDIX}

$\mathbf{M}=\left[\mathbf{M}_{j n}\right], \mathbf{M}_{j n}=\delta_{j n}+2 \hat{M} \hat{S}_{j n}$.

$\mathbf{K}_{1}=\left[\mathrm{K}_{1 j n}\right], \mathrm{K}_{1 j n}=4 \hat{M} \dot{\xi} R_{j n}$.

$\mathbf{K}_{2}=\left[\mathrm{K}_{2 j n}\right]$,

$\mathrm{K}_{2 j n}=\omega_{j}^{2} \delta_{j n}+2 \hat{M}\left(\dot{\zeta} S_{j n}-\mu \hat{g} R_{j n} v_{0}\right)$.

$\overline{\mathbf{h}}=\left(\bar{h}_{j}\right), \bar{h}_{j}=2 \hat{M}\left(\hat{g} \hat{S}_{j}+\dot{\xi}^{2} S_{j 1} v_{0}\right)$.

$\overline{\mathbf{B}}=\left[\overline{\mathrm{B}}_{j k}\right], \overline{\mathbf{B}}_{j k}=-\hat{S}_{j}\left(\eta_{k}\right)$.

$\overline{\mathbf{u}}=\left(\hat{u}_{k}\right)$. 